\title{
Complementary Eigenvalue Problem in systems with frictional contact: the Stiffness Matrix for the contact nodes between different materials
}

\author{
Maria Antónia Forjaz \\ Center of Mathematics \\ University of Minho \\ Email: maf@math.uminho.pt
}

\author{
António Mário Almeida \\ and T. de Lacerda-Arôso \\ Department of Physics \\ University of Minho \\ Email: coimbra@fisica.uminho.pt \\ tla@fisica.uminho.pt
}

\author{
Jorge Pamplona \\ CIGR \\ University of Minho \\ Email: jopamp@dct.uminho.pt
}

\begin{abstract}
This work addresses a numerical study of static equilibrium states of finite dimensional systems with frictional contact and its application to the particular problem of friction between two geological layers with different viscosity. Its formulation as a complementarity eigenproblem requires the building up of mass $M$ and stiffness $K$ matrices to solve the eigenvalue equations for the relative deformation of two contacting materials.
\end{abstract}

Keywords: Finite Element Method, frictional contact, stiffness matrix, Complementary Eigenvalue Problem.

\section{INTRODUCTION}

The classical Eigenvalue Problem finds important applications in different areas of economics, science and engineering [1]. In Physics its applications are beyond mention for so wide and important. Particularly, in the typical models employed in condensed matter physics which are based in the oscillator paradigm, vibrations are everywhere and so are the eigenvalues associated with them [2]. In fact, it is in the dynamic behavior of a physical system that the eigenvalue problems are best known.

The study of instabilities and bifurcations in systems with friction has been motivated by many experimental observations related to technological problems or industrial processes. In [3] and [4] propose a mixed explicit complementarity eigenproblem, equivalent to a mixed complementarity-inclusion eigenproblem, and also to a set of classical generalized linear eigenproblems.

This work is presented according to: Section I, which contains the mathematical definitions for the complementary eigenproblem; Section II where the formulation of finite dimensional frictional contact problem for different material nodes is studied and the crucial stiffness matrix for this situation is established; Section III includes the procedure to formulate the frictional contact issue as a Complementary Eigenvalue Problem.

\section{A. Eigenproblem}

The classical Eigenvalue Problem (EP) consists of finding a scalar $\lambda$ (eigenvalue) and a non-zero vector $x$ (eigenvector associated to the eigenvalue $\lambda$ ) such that

$$
A x=\lambda x,
$$

where $A$ is a square, complex or real, matrix.

In some circumstances, considering $A$ symmetric, it is advantageous to use an optimization approach to the Rayleigh quotient, $R_{A}(x)$, [3]

$$
R_{A}(x):=\frac{x^{T} A x}{x^{T} x}
$$

In fact, since $x \neq 0$, the gradient of the Rayleigh quotient is given by

$$
\nabla R_{A}(x):=\frac{2}{x^{T} x}\left(A-R_{A}(x) I\right) x,
$$

and the (non-zero) critical points of $R_{A}(x)$ are precisely the eigenvectors of $A$, and hence the critical points corresponding to a given eigenvalues form a space whose dimension is equal to the dimension of this eigenvalue. The critical points corresponding to the largest (respectively smallest) eigenvalue are global maxima (respectively minima) of $R_{A}(x)$.

Given two matrices $A, B \in \mathbb{R}^{n \times n}$, the problem of finding a scalar $\lambda \in \mathbb{R}$ and a non-vector $x \in \mathbb{R}^{n}$ such that

$$
A x=\lambda B x .
$$

is often referred as the Generalized Eigenvalue Problem (GEP).

\section{B. The Complementary Eigenproblem}

The Eigenvalue Complementary Problem (EiCP) is an extension of EP, first defined in [3], [5]. For a given matrix $A \in \mathbb{R}^{n \times n}$ and a positive matrix $B \in \mathbb{R}^{n \times n}$ (i.e. $x^{T} B x>$ 
$0, \forall x \neq 0$ ), the EiCP consists on finding $\lambda \in \mathbb{R}^{+}$, and the two vectors $x \in \mathbb{R}^{n}$ and $w \in \mathbb{R}^{n}$ such that

$$
\left\{\begin{array}{l}
w=(\lambda B-A) x \\
w \geq 0, x \geq 0 \\
x^{T} w=0 \\
e^{T} x=1
\end{array}\right.
$$

where $e \in \mathbb{R}^{n}$ is a vector of ones. The last constraint guarantees that the null vector $x$ is not a solution to the problem.

The EiCP differs from the classical Eigenvalue Problem (EP) on the existence of nonnegative constraints on its variables and complementarity constraints between pairs of variables.

The EiCP can be formulated as a Variational Inequality Problem (VI) on the simplex

$$
\Omega=\left\{x \in \mathbb{R}^{n}: e^{T} x=1, x \geq 0\right\}
$$

and, since the VI on the simplex has a solution, the same is true for the EiCP [6].

EiCP is called symmetric if the matrices $A$ and $B$ are both symmetries, and $B$ is positive definite, and in this case EiCP is equivalent to the problem of finding a stationary point of the Rayleigh function on the simplex [3].

Different algorithms have been developed in order to find a complementary eigenvalue and the associated eigenvector [7], [8], [9], [10] or, more recently, to find all the complementary eigenvalues [11].

\section{The Mixed Complementary Eigenvalue Problem}

The EiCP is a particular case of the so-called Mixed Complementary Eigenvalue Problem (MEiCP) that consistes on finding $\lambda \in \mathbb{R}^{+}$, and the non-zero vector $x \in \mathbb{R}^{n}$ and the vector $w \in \mathbb{R}^{n}$ such that

$$
\left\{\begin{array}{l}
w=(\lambda B-A) x \\
w_{J} \geq 0, x_{J} \geq 0 \\
x_{J}^{T} w_{J}=0 \\
e^{T} x=1
\end{array}\right.
$$

where $e \in \mathbb{R}^{n}$ is a vector of ones, $x_{J} \equiv\left(x_{j}, j \in J\right), w_{J} \equiv$ $\left(w_{j}, j \in J\right), J \subseteq\{1, \ldots, n\}$ and $\bar{J} \subseteq\{1, \ldots, n\} \backslash J$. Observe that the EiCP is for $J=\{1, \ldots, n\}$.

\section{SySTEMS WITH FRICTIONAL CONTACT: THE STIFFNESS MATRIX}

In the present work we build up the stiffness matrix $\mathrm{K}$ at the frictional contact between two materials with different visco-elastic properties [12].

In geological processes (e.g. layering, folding and boudinage), materials with contrasting physical properties are often in contact. The comprehension of the contact dynamics of such two layers is the first step to solve relevant and more complex geological problems.

The herein problem consists on two materials, $A$ and $B$, with different mechanical properties (namely, density, viscosity, and stiffness) in direct contact. Each material is described by a two-dimensional mesh whose nodes are separated by distance $a$ and to which is assigned a fraction of the total mass (density $\times a^{3}$ ). Bonding between every two point masses is characterized by stiffness $k$ (which includes the relevant properties to the problem).

Actually, the net force $\mathbf{F}$ acting on the individual point masses relate to their respective displacements $\mathbf{u}$ by

$$
\mathbf{F}=k \mathbf{u}
$$

In Figure 1, two layers of point masses are shown: round dots for material $A$ and squares for material $B$. Dashed lines depict the interactions amid them. The particular connections between neighbouring point masses belonging to either one or the other material are coloured in black.

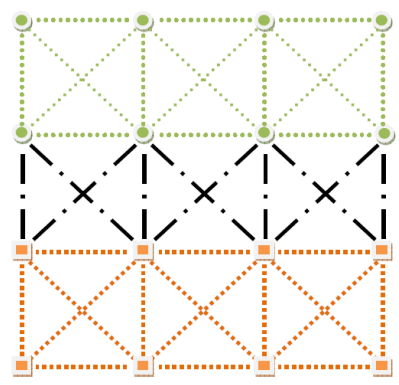

Fig. 1. A model of materials $A$ and $B$ in contact.

A 4-mass cell consisting of two point masses of the lower level $A$ and two other of the upper material $B$ is outlined in Figure 2 with its nodal forces and displacements.

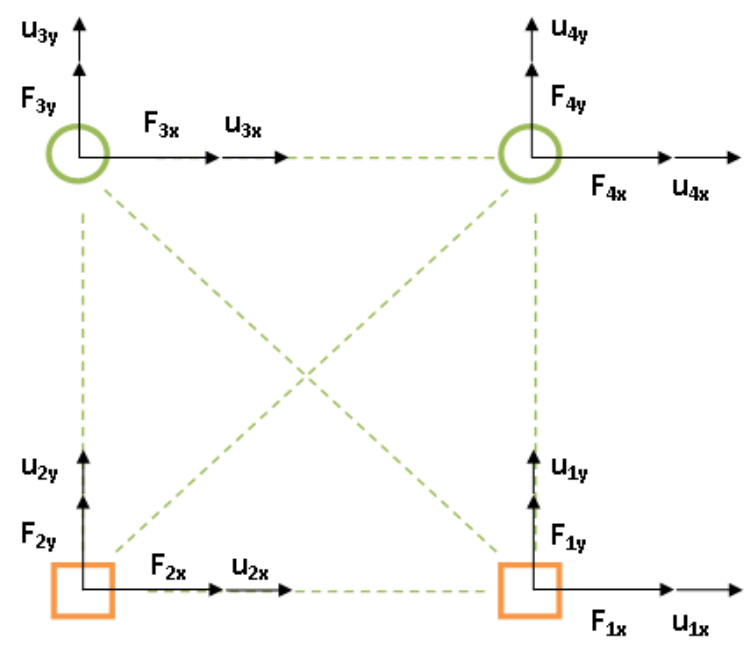

Fig. 2. Applied forces and displacement at each node.

A stiffness matrix is to be built up for those 4 point masses and interactions. For bonding between adjoining points belonging to the same layer, we take the known properties of that material to define $k$. In every likelihood, these materials are isotropic and homogeneous and so we assume $k$ to be constant - $k_{A}$ for material $A$ and $k_{B}$ for material $B$. When addressing interactions between point masses of different materials, one must take either the average of $k_{A}$ and $k_{B}$, or evaluate the 
$k_{A B}$ suitable for the situation, possibly by using empirical data, when available.

$$
\left(\begin{array}{l}
F_{1 x} \\
F_{1 y} \\
F_{2 x} \\
F_{2 y} \\
F_{3 x} \\
F_{3 y} \\
F_{4 x} \\
F_{4 y}
\end{array}\right)=\mathrm{K}\left(\begin{array}{l}
u_{1 x} \\
u_{1 y} \\
u_{2 x} \\
u_{2 y} \\
u_{3 x} \\
u_{3 y} \\
u_{4 x} \\
u_{4 y}
\end{array}\right)
$$

For the subset of point masses 1,2, 3 and 4 we define the angles $\alpha, \beta, \gamma$ and $\theta$, as shown in Figure 3 .

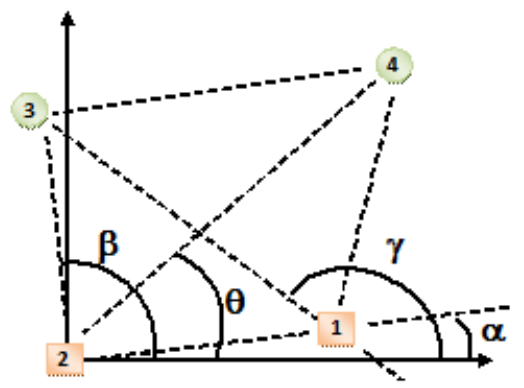

Fig. 3. Deformation at the contact of the two materials.

The $\mathrm{K}$ matrix that encompass all the nodes in the structure results from rightfully adding the four individual stiffness matrices

$$
A_{i}=\left(\begin{array}{cc}
c_{i}^{2} & c s_{i} \\
c s_{i} & s_{i}^{2}
\end{array}\right)
$$

of each point mass. The elements of these matrices represent the projection of the force and respective displacement along the directions $\mathrm{X}$ and $\mathrm{Y}$, according to the angles $\alpha, \beta, \gamma$ and $\theta$ (see Figure 3). (We use the short notation $c_{i}=\cos i, s_{i}=\sin i$, $c s_{i}=\cos i \sin i$.)

Thus, the global stiffness matrix writes

$$
\mathrm{K}=k_{A B}\left(\begin{array}{ccccc}
P & \frac{k_{A}}{k_{A B}} A_{\alpha} & \mid & A_{\gamma} & A_{\beta} \\
\frac{k_{A}}{k_{A B}} A_{\alpha} & Q & \mid & A_{\beta} & A_{\theta} \\
-- & -- & -- & -- & -- \\
A_{\gamma} & A_{\beta} & \mid & R & \frac{k_{B}}{k_{A B}} A_{\alpha} \\
A_{\beta} & A_{\theta} & \mid & \frac{k_{B}}{k_{A B}} A_{\alpha} & S
\end{array}\right)
$$

Each block $P, Q, R$ and $S$ traduces the global stiffness related to each node with contributions, as appropriate, by each neighbour node.

$$
P=\left(\begin{array}{cc}
\frac{k_{A}}{k_{A B}} c_{\alpha}^{2}+c_{\beta}^{2}+c_{\gamma}^{2} & \frac{k_{A}}{k_{A B}} c s_{\alpha}+c s_{\beta}+c s_{\gamma} \\
\frac{k_{A}}{k_{A B}} c s_{\alpha}+c s_{\beta}+c s_{\gamma} & \frac{k_{A}}{k_{A B}} s_{\alpha}^{2}+s_{\beta}^{2}+s_{\gamma}^{2}
\end{array}\right)
$$

$$
\begin{aligned}
& Q=\left(\begin{array}{cc}
\frac{k_{A}}{k_{A B}} c_{\alpha}^{2}+c_{\beta}^{2}+c_{\theta}^{2} & \frac{k_{A}}{k_{A B}} c s_{\alpha}+c s_{\beta}+c s_{\theta} \\
\frac{k_{A}}{k_{A B}} c s_{\alpha}+c s_{\beta}+c s_{\theta} & \frac{k_{A}}{k_{A B}} s_{\alpha}^{2}+s_{\beta}^{2}+s_{\theta}^{2}
\end{array}\right) \\
& R=\left(\begin{array}{cc}
\frac{k_{B}}{k_{A B}} c_{\alpha}^{2}+c_{\beta}^{2}+c_{\gamma}^{2} & \frac{k_{B}}{k_{A B}} c s_{\alpha}+c s_{\beta}+c s_{\gamma} \\
\frac{k_{B}}{k_{A B}} c s_{\alpha}+c s_{\beta}+c s_{\gamma} & \frac{k_{B}}{k_{A B}} s_{\alpha}^{2}+s_{\beta}^{2}+s_{\gamma}^{2}
\end{array}\right) \\
& S=\left(\begin{array}{cc}
\frac{k_{B}}{k_{A B}} c_{\alpha}^{2}+c_{\beta}^{2}+c_{\gamma}^{2} & \frac{k_{B}}{k_{A B}} c s_{\alpha}+c s_{\beta}+c s_{\gamma} \\
\frac{k_{B}}{k_{A B}} c s_{\alpha}+c s_{\beta}+c s_{\gamma} & \frac{k_{B}}{k_{A B}} s_{\alpha}^{2}+s_{\beta}^{2}+s_{\gamma}^{2}
\end{array}\right)
\end{aligned}
$$

The four blocks placed in the upper-left position relate to the $A$-material and the connections between the two materials, since they only contemplate $1-2$-type bonds and $A$ - $B(1-4$, $2-3,1-3,2-4)$ bonds. Else, the four blocks placed in the down-right position relate to the sole B and A-B bonds. For such reason, in the former case only $k_{A}$ and $k_{A B}$ parameters are contemplated while in the latter $k_{B}$ and $k_{A B}$ parameters appear.

\section{Complementary Eigenvalue Problems}

Under constant applied forces $F^{0}$ the dynamics of the whole mass system is governed by momentum balance equations

$$
M \ddot{u}(t)+K u(t)=F^{0}+R(t)
$$

where $M$ is the mass matrix (symmetric and positive definite) and $K$ is the stiffness matrix (positive definite) and $R(t)$ denotes the reaction forces at time $t(t \geq 0)$,

Under the same applied forces $F^{0}$ for a constant displacement rate ( $\ddot{u}=0)$, equation (6) assumes the form

$$
K u^{0}=F^{0}+R^{0}
$$

In [13] it is shown that for some $t$ there are dynamic solutions of the form

$$
\begin{array}{r}
u(t)=u^{0}+\alpha(t) v \\
R(t)=R^{0}+\beta(t) w
\end{array}
$$

if and only if there exists a number $\lambda \geq 0$, and two vectors $v$ and $w(v \neq 0)$, are such that

$$
\begin{aligned}
\left(\lambda^{2} M+K\right) v & =w \\
w_{j} & =0 \\
v_{d} & =0
\end{aligned}
$$

Note that $v$ and $w$ define constant directions in the sets of right admissible displacement and reaction rates at the equilibrium state $\left(u^{0}, R^{0}\right)$, the function of time $\alpha$ is twice continuously differentiable, $\alpha$ and $\dot{\alpha}$ are non-negative and non-decreasing in $[\tau, \tau+\triangle \tau[$, the function $\beta$ is continuous, non-negative and nondecreasing in the same interval, and the initial values $\alpha(\tau) \geq 0$, $\dot{\alpha}(\tau) \geq 0$ are arbitrarily small.

The problem is subsequently transformed into a nonmonotone mixed complementarity problem (MEiCP), in which 
the unknown eigenvalue is treated as a non-negative variable that is complementary with an additional variable involved in a normalising constraint that prevents the trivial solution, stated as it is in equation (3).

\section{FUTURE WORK}

The method of MEiCP is to be applied to extensive layers of different materials in contact and under intense strain in order to study the deformation at the contact surface.

Latter on we aim to build up the stiffness matrix of an high viscosity layer embedded in a low viscosity one, which leads to deformation.

\section{ACKNOWLEDGMENT}

The first author research was supported by the Research Centre of Mathematics of the University of Minho with the Portuguese Funds from the Fundação para a Ciência e a Tecnologia, through the Project PEstOE/MAT/UI0013/2014

\section{REFERENCES}

[1] F. Chatelin, Eigenvalues of Matrices, ser. Classics in Applied Mathematics. New York: Wiley, 1993.

[2] B. N. Parlett, The symmetric eigenvalue problem, ser. Classics in Applied Mathematics. Philadelphia: S.I.A.M, 1998.

[3] J. J. Júdice, C. Humes, and M. Queiroz, "The symmetric eigenvalue complementarity problem," Mathematics of Computation, vol. 73, no. 1, pp. 1849-1863, January 2004.

[4] A. Pinto da Costa, I. Figueiredo, J. J. Júdice, and J. Martins, "A complementarity eigenproblem in the stability analysis of finite dimensional elastic systems with frictional contact," in Complementarity: Applications, Algorithms and Extensions, M. Ferris, O. Mangasarian, and J. Pang, Eds. Dordrecht: Kluwer Academic Publishers, 2001, pp. 67-83.

[5] A. Seeger, "Eigenvalue analysis of equilibrium processes defined by linear complementarity conditions," Linear Algebra and its Applications, vol. 292, no. 1-3, pp. 1-14, May 1999.

[6] J. J. Júdice, H. Sherali, and I. Ribeiro, "On the asymmetric eigenvalue complementarity problem," Optimization Methods and Software, vol. 24, no. 1, pp. 549-586, January 2009.

[7] J. J. Judice, H. Sherali, and I. Ribeiro, "The eigenvalue complementarity problem," Computational Optimization and Applications, vol. 37, no. 1, pp. 139-156, January 2007.

[8] J. J. Júdice and M. Raydan, "On the solution of the symmetric eigenvalue complementarity problem by the spectral projected gradient algorithm," Numerical Algorithms, vol. 45, no. 1, pp. 391-407, January 2008 .

[9] A. P. da Costa and A. Seeger, "Cone-constrained eigenvalue problems: theory and algorithms." Comp. Opt. and Appl., vol. 45, no. 1, pp. 25-57, 2010

[10] M. M. Le Thi, T. D. Pham Dinh, and J. J. Júdice, "A dc programming approach for solving the symmetric eigenvalue complementarity problem," Computational Optimization and Applications, vol. 51, no. 3, pp. 1097-1117, April 2012.

[11] L. M. Fernandes, J. J. Júdice, H. Sherali, and M. F. Forjaz, "On an enumerative algorithm for solving eigenvalue complementarity problems,' Computational Optimization and Applications, vol. 1, no. 1, pp. 1-22, January 2013.

[12] C. Felippa, "A historical outline of matrix structural analysis: A play in three acts," Computers \& Structures, vol. 79, pp. 1313-1324, 2001.

[13] J. Martins, S. Barbarin, M. Raous, and A. Pinto da Costa, "Dynamic stability of finite dimensional linearly elastic systems with unilateral contact and coulomb friction," Computer Methods in Applied Mechanics and Engineering, vol. 177, no. 1, pp. 289-328, January 1999.
[14] J. J. Júdice, A. Pinto da Costa, J. Martins, and I. Figueiredo, "The directional instability problem in systems with frictional contacts," Computer Methods in Applied Mechanics and Engineering, vol. 193, no. 1, pp. 357-384, January 2004. 